Service social

\title{
L'évaluateur dans un contexte institutionnel : proposition d'un code de déontologie
}

\section{Jean-Pierre Bolduc et Jacques Plante}

Volume 40, numéro 1, 1991

Éthique et intervention sociale

URI : https://id.erudit.org/iderudit/706516ar

DOI : https://doi.org/10.7202/706516ar

Aller au sommaire du numéro

Éditeur(s)

École de service social de l'Université Laval

ISSN

1708-1734 (numérique)

Découvrir la revue

Citer cet article

Bolduc, J.-P. \& Plante, J. (1991). L'évaluateur dans un contexte institutionnel : proposition d'un code de déontologie. Service social, 40(1), 91-124.

https://doi.org/10.7202/706516ar
Résumé de l'article

La conduite d'une évaluation va bien au-delà de l'utilisation de modèles méthodologiques. Elle demande à l'évaluateur de créer des conditions de réalisation favorables et d'adopter une démarche professionnelle basée sur un modèle de conduite où l'éthique et la déontologie supportent la réalisation de l'évaluation. Dans cet article, les auteurs font le point sur les principaux aspects pouvant influencer la conduite d'une évaluation, sur la nécessité de disposer d'un référentiel de conduite professionnel, afin de réduire et de résoudre les problèmes éthiques, et, finalement, ils proposent un code de déontologie pour l'évaluateur. 
Jean-Pierre Bolduc, professeur au Département de nutrition humaine et consommation, Faculté des sciences de l'agriculture et de l'alimentation, Université Laval.

Jacques Plante, professeur au Département de mesure et évaluation, Faculté des sciences de l'éducation, Université Laval.

\section{L'évaluateur dans un contexte institutionnel : proposition d'un code de déontologie}

Jean-Pierre Bolduc Jacques Plante

\section{Partie I \\ L'évaluateur dans un contexte institutionnel}

\section{L'éthique et la déontologie en évaluation institutionnelle : qu'en est-il ?}

En éducation, santé et bien-être, il est étonnant et même regrettable de constater dans les ouvrages liés à l'évaluation que peu de spécialistes du domaine se sont penchés explicitement sur le thème de l'éthique. Généralement et encore aujourd'hui, plusieurs auteurs se contentent de laisser deviner quelques inquiétudes à ce sujet. Plusieurs modèles d'évaluation ont été développés et malgré leur influence évidente sur le comportement de l'évaluateur, nous n'y trouvons pas les balises suffisantes permettant au spécialiste d'adopter une ligne et des règles de conduite qui débouchent sur un professionnalisme où l'éthique et la déontologie supportent la réalisation de l'évaluation.

Dans les faits, un évaluateur trouve difficilement les informations et la documentation concernant cet aspect important de sa vie professionnelle. Nous tenterons dans le présent article de remédier quelque peu à cette lacune en apportant, au lecteur ou à la lectrice, des ren- 
seignements susceptibles d'aider le spécialiste en évaluation, ou toute autre personne chargée de procéder à une évaluation, à exercer sa profession avec toute la responsabilité qui lui incombe. Nous nous proposons également, en tenant compte des conditions inhérentes à une évaluation éthique, de présenter un code de déontologie susceptible d'assister le spécialiste en évaluation dans l'exercice de sa profession.

\section{Profession : spécialiste en évaluation}

Être "évaluateur " dans un contexte institutionnel, en éducation, santé, bien-être, consiste à exercer une profession relativement nouvelle, en pleine expansion, et qui se situe dans la famille des professions de management (Windle et Neigher, 1978; Ingle et Klauss, 1980; Stanfield et Smith, 1984). De ce fait, et afin qu'elle ne soit pas perçue comme une duplication de professions déjà existantes, il importe de préciser la nature et le champ d'activité de cette spécialité. Ainsi qu'il est défini dans la plupart des dictionnaires, le terme spécialiste désigne une personne possédant des connaissances théoriques et pratiques dans un domaine précis. Bien qu'il lui faille constamment faire appel à des notions provenant de multiples domaines pour mener sa tâche à terme, le spécialiste en évaluation possède plus spécifiquement des connaissances sur les procédures et les techniques d'investigation permettant de réaliser une évaluation. Comme l'évaluation " est un processus par lequel on délimite, obtient et fournit des informations utiles permettant de juger des décisions possibles» (Stufflebeam, $1980: 48$ ), l'évaluateur devient une personneressource ou un conseiller offrant des services professionnels qui servent à faciliter, voire même bonifier, la prise de décision à l'intérieur d'une institution. Son champ de travail est donc relativement vaste. II peut être amené à collaborer à l'évaluation d'une intervention individuelle ou de groupe, à l'évaluation d'un service, d'un programme ou même de l'institution tout entière. Cela peut concerner alors tout aussi bien le fonctionnement général de cette institution que le climat organisationnel, les personnels, les objectifs et orientations visés, etc. Dans ce contexte institutionnel, il exerce une profession où l'investigation et la production d'informations occupent une place prépondérante. Ses activités ont pour but de fournir aux responsables des données pertinentes facilitant la prise de décision selon des options et des critères préalablement déterminés.

L'évaluateur est alors appelé à travailler avec divers généralistes, spécialistes et preneurs de décisions afin de les aider $: 1$. à poser un diagnostic sur une situation quelconque, et 2 . à prendre des déci- 
sions, si nécessaire, sur les solutions possibles pour remédier à cette situation. Il agit ainsi comme professionnel de soutien au moment de l'évaluation et il exerce un rôle de complémentarité au regard des autres professionnels responsables d'un programme ou d'une institution. Il en résulte qu'en plus de se doter de règles de pratique qui lui sont propres, l'évaluateur doit constamment tenir compte de celles des autres professionnels qu'il côtoie.

\section{L'éthique et la déontologie : définitions}

Étymologiquement, le mot éthique vient du nom grec êthos qui signifie "mœurs » et de son qualificatif êthikos désignant le caractère moral d'une activité ou d'un comportement (Kibler et Van Hoose, 1981). Dans le vocabulaire contemporain, ce mot est fréquemment utilisé comme épithète pour signifier l'appartenance à une morale reconnue et acceptée par une collectivité. On parle alors d'une science éthique, d'un comportement éthique, d'une évaluation éthique, etc. Cependant, l'utilisation de ce mot dépasse le simple rôle d'attribut. II peut aussi être utilisé pour désigner la science qui traite de la morale (philosophiquement, la morale constitue l'ensemble des valeurs et des règles de conduite reconnues comme normes dans une société). Cette discipline renvoie donc au sens moral individuel ou collectif dans la conduite d'une personne, d'un groupe ou encore d'un organisme au cours du déroulement d'une quelconque activité.

La déontologie quant à elle se distingue légèrement de l'éthique. L'origine de ce terme vient de la fusion des mots grecs deon, ontos et logos qui signifient respectivement "ce qu'il faut faire " et " discours ". La déontologie se définit donc comme étant la science qui s'intéresse aux devoirs à remplir. Tout comme l'éthique, la déontologie fait appel au sens moral d'un individu ou d'un groupe de personnes, mais elle englobe en plus l'aspect pratique, la façon de procéder dans l'exécution d'une tâche. Dans le langage usuel, l'éthique et la déontologie se trouvent donc fortement associées, voire confondues. Habituellement, la déontologie se révèle être une résultante de l'éthique et du sens moral cité précédemment. Toutefois, certaines situations montrent qu'il n'en est pas toujours ainsi et que l'utilisation indistincte de ces deux mots devrait être évitée. Pour mieux saisir cela, prenons l'exemple suivant : si nous demandions à un criminel de nous décrire ce qu'il faut faire pour être reconnu comme un membre à part entière dans son milieu, il nous parlerait des règles de pratique de son métier, c'est-à-dire de la déontologie reconnue dans ce milieu. Ce que nous aurions à faire par la suite serait de vérifier si toutes ces choses relèvent du domaine de la morale, de l'éthique! 


\section{Conditions préalables à la réalisation d'une évaluation éthique}

Dans leur collaboration à l'article de Kottler (1982), Paradise et Siegelwaks relèvent deux aspects importants à considérer pour établir le lien entre une démarche professionnelle et l'élaboration d'un modèle de conduite :

- les actes professionnels ou l'aspect pratique de l'exercice d'une profession, et

- les rapports entre les personnes ou l'aspect philosophique de la profession.

La constante interaction entre ces deux aspects trouve tout son sens dans la définition des règles et des devoirs qui serviront à déterminer des standards à inclure dans un modèle que l'on veut garant de la conduite et de la réputation du professionnel qui s'y conforme. Du point de vue de l'évaluation, ces deux aspects peuvent permettre d'éviter les problèmes éthiques qui proviennent souvent d'une mauvaise connaissance des particularités du milieu où s'effectue l'évaluation. Adelman (1980) estime qu'au tout début de la démarche, ce spécialiste devrait se considérer beaucoup plus comme un " anthropologue-sociologue " qu'un évaluateur. En effet, pour mener à terme une évaluation sans affronter trop d'embûches, il apparaît primordial de tracer un portrait fidèle du milieu et du contexte dans lequel se déroulera l'évaluation. L'évaluateur peut ainsi s'appuyer sur de solides assises pour entreprendre sa démarche.

Dans son analyse, Clem. Adelman s'attarde surtout aux multiples aspects pouvant influencer la réalisation d'une évaluation et à l'émergence de différentes sortes de problèmes ayant des conséquences sur l'éthique. II mentionne que le contexte de l'évaluation est principalement déterminé par divers facteurs comme : les attentes des gens du milieu, la résistance aux changements qu'ils démontrent, la menace que peut représenter une évaluation, l'accessibilité aux ressources tant financières, humaines que temporelles, les particularités culturelles du milieu et quelques autres points à voir ultérieurement. Pour l'auteur, l'ensemble de ces facteurs constitue un amalgame d'aspects indissociables qu'il appelle "le champ d'intervention ». De plus, il précise que la conduite d'une évaluation est en grande partie fonction de la faculté d'anticipation de l'évaluateur face aux problèmes potentiels. II suggère donc à ce dernier de faire une étude en profondeur du champ d'intervention avant même d'accepter l'offre qui lui est faite. Cela permettra d'apporter, en temps et lieu, des réponses précises aux interrogations des gens du milieu et de réduire ainsi quelques sources de problèmes. 


\section{L'évaluateur, le mandant et les auditoires cibles}

Parallèlement à cette analyse des particularités du milieu, Merwin et Weiner (1985) suggèrent fortement à l'évaluateur de définir quelles sont les personnes touchées ou concernées par l'évaluation. En se référant à divers écrits dont ceux de Stufflebeam, ces auteurs dénombrent trois grandes catégories : 1) les spécialistes de l'évaluation, 2) les généralistes de l'évaluation, et 3) les groupes de personnes visés par le champ d'investigation. Pour eux, il est primordial de procéder à cet exercice pour clarifier le rôle de chacun et ainsi favoriser un bon déroulement de toute l'évaluation. De plus, cela permettra à l'évaluateur de connaître les préoccupations de chacun à l'égard de la démarche entreprise et $d^{\prime}$ 'ainsi réduire les sources de tension pouvant surgir entre ces différentes catégories d'individus au moment de l'évaluation.

La catégorie "spécialistes de l'évaluation " regroupe tous les intervenants spécialisés dans la conduite d'une évaluation, comme un évaluateur ou une agence œuvrant dans ce domaine. Pour sa part, la catégorie "généralistes de l'évaluation " rassemble les gestionnaires, les personnes responsables d'un programme, d'un service ou d'une institution et tous ceux qui fournissent une expertise au moment de l'évaluation (même si ces derniers peuvent être reconnus comme des spécialistes dans un autre domaine). Ces individus sont aussi fréquemment appelés les décideurs ou encore "le mandant » de l'évaluation. Finalement, la troisième catégorie couvre l'ensemble des autres groupes engagés dans l'évaluation. Une nouvelle appellation semble se définir pour désigner cette catégorie d'individus : "les auditoires cibles ». Ces auditoires regroupent toutes les personnes ou tous les groupes de personnes gravitant autour d'un programme ou $\mathrm{d}^{\prime}$ une institution. Bref, trois catégories de personnes ayant des rôles différents à jouer au cours de la démarche évaluative. Dans chaque cas, la perception de l'évaluation risque d'être fort différente et les problèmes qui en résultent iront de pair. En conséquence, il appert que l'évaluateur se doit de faire préciser par son mandant la nature exacte des services pour lesquels on le sollicite. Cette mise au point devrait évidemment se faire par le moyen d'une entente conclue entre les parties avant qu'elles s'engagent dans le processus évaluatif.

\section{Conduite de l'évaluation}

La documentation sur ce thème abonde mais, curieusement, les auteurs ont bien du mal à établir un consensus. La raison à l'origine de plusieurs désaccords sur la conduite de l'évaluation vient surtout du fait que les auteurs accordent beaucoup plus d'importance à la mé- 
thodologie et au développement de modèles qu'à tout autre aspect. En conséquence, deux grandes écoles de pensée sont apparues en évaluation : I'approche formaliste et l'approche naturaliste. Au lieu de prendre position sur ce sujet de la façon employée par nombre d'auteurs, nous utiliserons une autre perspective pour parler de la conduite de l'évaluation : I'approche éthique. Précisons tout de suite que le but ici visé n'est pas de lancer une nouvelle école de pensée en évaluation et encore moins de laisser sous-entendre que les approches naturaliste et formaliste ne font pas preuve d'éthique. Bien au contraire, chacune apporte des précisions intéressantes et valables tout en donnant aux évaluateurs la possibilité de choisir une méthode pertinente et adaptée au contexte d'ensemble de l'évaluation. Avant tout, nous pensons qu'un spécialiste de ce domaine, en éducation, santé et bien-être, devrait accorder priorité à l'éthique pour ensuite choisir la méthode qui convient le mieux à la situation.

\section{Rôle de l'évaluateur, contextes méthodologiques et résistances}

Au cours de ses travaux, Lincoln (1986) a dénombré quatre générations d'évaluateurs : 1) le technicien, 2) le planificateur, 3) l'évaluateur-juge, et 4) I'animateur-conseil. D'après cette auteure, chacune de ces générations d'évaluateurs tente d'apporter une réponse aux critiques adressées à la génération précédente tout en assumant ce qu'elle était. Bien qu'il serait intéressant de parler en détail de chaque génération, la dernière mérite plus particulièrement notre attention.

Contrairement aux générations précédentes, l'évaluateur de la quatrième génération n'est plus considéré comme un spécialiste qui influe sur la prise de décision, ni comme quelqu'un qui va juger si une chose est bonne ou mauvaise dans un contexte donné. Son rôle consiste essentiellement à agir dans le respect des besoins du milieu en donnant des conseils et des avis susceptibles de favoriser la conduite de l'évaluation. La prise de décision sur quelque point que ce soit se fera donc par les gens du milieu à partir de critères reposant sur leurs propres valeurs. L'évaluateur se veut, dans ce contexte, un animateur-conseil qui fournit une expertise conforme à la morale sociale du milieu et aux attentes des personnes intéressées.

Comme le souligne Shapiro (1985), "There are three contexts within which evaluators operate : an ethical context, an organizational context, and a political context » (p. 245). Bien que chacun de ces contextes puisse être abordé individuellement, I'auteur insiste sur le fait qu'ils sont interdépendants et que, dans la pratique, il n'est pas simple de les dissocier. La raison : ces trois contextes relèvent de la 
méthodologie, c'est-à-dire de la manière de faire, de procéder. En résumé, l'identification et la mise en application d'un modèle ou d'une méthodologie d'évaluation dépendent de ces trois contextes. À la fin, l'évaluateur doit se préoccuper autant de l'aspect éthique de l'évaluation que de son organisation, tout en s'intéressant au contexte politique. Il doit faire prendre conscience à tous ceux qui collaborent à l'activité de l'importance de ces aspects dans la réalisation de l'évaluation. L'omission ou la négligence de l'un par rapport aux autres risque parfois de faire surgir des malentendus difficiles à régler en cours d'évaluation. Bref, ces trois aspects doivent être considérés simultanément par les divers intervenants (participants, décideurs et évaluateur) afin d'établir un plan d'intervention opérationnel, efficace et respectant les attentes des gens du milieu.

Avant d'entreprendre la réalisation d'une évaluation, l'évaluateur doit aussi considérer un autre important aspect pouvant menacer la conduite de celle-ci. II lui faut reconnaître les résistances rencontrées chez les participants et dans le milieu, pour ensuite essayer de les atténuer. D'après Kilburg (1980), la nature de ces résistances peut être psychologique ou philosophique. Elles se décèlent chez un participant, chez un groupe de personnes ou encore chez des professionnels appelés à collaborer à l'activité. De plus, ces résistances s'expriment sous une forme active ou passive. L'évaluateur doit donc être attentif à leurs manifestations et adopter différentes stratégies pour contrer, à court ou à moyen terme, les effets négatifs sur l'évaluation.

Jusqu'ici nous avons délibérément abordé le thème " conditions préalables à la réalisation d'une évaluation éthique " dans une perspective très générale. De ce fait, il nous faut souligner que les points qui viennent d'être soulevés ne représentent pas l'ensemble des conditions préalables à respecter pour la réalisation d'une évaluation éthique. En effet, chaque évaluation possède des caractéristiques qui lui sont propres et les situations favorables à l'émergence de problèmes éthiques sont fonction de ces caractéristiques. II revient donc à l'évaluateur d'identifier ces situations particulières et d'adopter tant des attitudes que des stratégies permettant d'y apporter une solution.

\section{L'évaluateur et le mandat}

Accepter un mandat va bien au-delà de la simple participation à une activité pour un professionnel. Cela exige de sa part une implication constante et un engagement quasi sans limite pour mener à terme l'opération entreprise. Parfois même, sa responsabilité civile, quand ce n'est pas tout simplement sa réputation, peut être mise en 
jeu. II apparaît donc primordial pour un évaluateur et pour son client d'établir contractuellement le cadre de toute entente ainsi que l'ensemble des conditions auxquelles ils conviennent de se soumettre. La rédaction de ce contrat devrait idéalement s'effectuer parallèlement et de façon quasi concomitante à l'analyse des facteurs pouvant influencer le déroulement de l'évaluation. Cela devrait permettre d'éliminer a priori bien des sources de mésententes entre les parties. Certes, il est utopique de penser que tout peut être prévu mais, comme le souligne Adelman (1980), certaines dispositions à inscrire au contrat relèvent de la plus élémentaire prudence et surtout de la plus fondamentale démarche professionnelle qu'il qualifie d'éthique.

\section{Services professionnels et expertise}

L'identification et la description des services offerts par le professionnel constituent des points à mettre au clair entre tous les intervenants participant à l'évaluation. Le soutien technique et matériel offert par l'évaluateur ainsi que sa collaboration à l'activité comportent des limites devant être déterminées dès que les parties entrent en contact. En effet, ce spécialiste n'étant pas omniscient ni omniprésent, il apparaît souhaitable de prévoir, même si la situation ne l'exige pas toujours, des dispositions au contrat concernant : a) la responsabilité et les obligations de l'évaluateur envers son mandant, et $b$ ) la collaboration d'autres professionnels offrant des services et une expertise ne relevant pas de la compétence de l'évaluateur. En agissant ainsi, l'évaluateur et le mandant seront à même de déterminer à tout moment les engagements pris l'un envers l'autre quant aux services à fournir pour effectuer l'évaluation.

Selon Ingle et Klauss (1980), l'expertise que fournit un évaluateur à son client se définit par les compétences que doit posséder ce professionnel. Ces dernières se répartissent en quatre champs d'application : 1) les compétences d'ordre technique, 2) les compétences liées à l'analyse politique d'un programme ou d'une institution, 3) les compétences d'ordre administratif, et 4) les compétences personnelles et interpersonnelles. Par conséquent, l'expertise exigée par le client peut faire appel à une, à plusieurs ou à l'ensemble de ces champs de compétences.

La catégorie "compétences techniques»englobe tout ce qui concerne la collecte et le traitement de l'information. L'expertise de l'évaluateur dans cette catégorie fait alors référence à son rôle de technicien de la mesure. Elle comprend l'organisation des procédures et des techniques de mesure, la collecte des données, la saisie (informatique) et le traitement statistique de ces dernières, l'analyse 
et l'interprétation des résultats, la production de rapports écrits et l'estimation financière des coûts de l'évaluation. L'évaluateur fournit donc une expertise purement technique sur la réalisation de l'évaluation.

Globalement, l'analyse politique relève de l'approche philosophique de l'évaluation. L'expertise de l'évaluateur fait alors appel à son rôle d'analyste de la situation dans son ensemble. Que ce soit pour un programme, un service ou une institution, l'évaluateur s'intéresse aux buts et aux objectifs poursuivis par le mandant, par la démarche évaluative, par l'objet d'évaluation et par les gens du milieu. De plus, il voit à articuler, clarifier et rendre accessibles les décisions prises avant, pendant ou après l'évaluation. De ce fait, il se doit d'être sensibilisé à tout le contexte " politique " de l'intervention et aux répercussions des décisions sur l'environnement.

D'après Ingle et Klauss, les compétences administratives sont probablement les plus négligées dans la formation d'un évaluateur. Pourtant, elles représentent un aspect important de l'expertise de ce spécialiste. Ces compétences portent essentiellement sur la gestion et le management. L'évaluateur doit posséder des compétences liées à la gestion budgétaire et financière, à la comptabilité, à la planification d'activités, à l'organisation des systèmes, à la négociation de contrats ou d'ententes et à toute autre pratique administrative courante. $D^{\prime}$ après les auteurs, un évaluateur ayant des faiblesses sur ce plan risque fort de s'embourber dans des procédures administratives pourtant essentielles à la compréhension de l'objet d'évaluation et à la conduite de cette dernière.

Finalement, les compétences personnelles/interpersonnelles se réfèrent aux qualités personnelles de l'évaluateur au regard de l'aspect "relations publiques" de ses fonctions. Ce professionnel doit posséder des habiletés et connaître différentes méthodes $d^{\prime}$ intervention sur le travail d'équipe, sur les relations publiques, sur la communication, sur la résolution de problèmes (de conflits), sur les styles de leadership, sur la direction de personnel et sur les interactions formelles et informelles qui existent entre les individus. Comme le soulignent Ingle et Klauss, cette expertise porte principalement sur les relations humaines entre les divers intervenants de l'évaluation. Cela demande donc à l'évaluateur d'être un expert des relations publiques. De plus, les multiples valeurs personnelles et collectives pouvant intervenir dans ces relations entre individus sont souvent source de problèmes ou de tensions au moment de l'évaluation. II faut donc que l'évaluateur ajuste son expertise aux valeurs préconisées par tous les intervenants du milieu afin d'établir un contexte favorable pour l'évaluation. 
Pour conclure sur ce sujet, mentionnons que l'évaluateur doit être plus qu'un expert de la collecte, du traitement et de l'interprétation d'informations. Les services professionnels pour lesquels on le sollicite exigent qu'il produise une expertise dans divers domaines. La conduite et le déroulement de l'évaluation demandent des connaissances et des habiletés que ce professionnel doit être en mesure $d^{\prime}$ offrir à son client. Cependant, malgré une certaine polyvalence de l'expertise de l'évaluateur, ce dernier ne peut à lui seul fournir toutes les réponses aux diverses situations et interrogations rencontrées au cours d'une évaluation. Alors, il devient primordial tant pour l'évaluateur que pour le mandant de prévoir, dans leur entente, la collaboration d'experts pouvant suppléer à ces exigences de l'évaluation.

\section{Aspects privés et publics d'un mandat}

Howe (1980) fait remarquer qu'une intervention professionnelle peut revêtir un caractère public ou privé. Les facteurs qui déterminent ce caractère sont le mandat donné au professionnel et la nature des services prodigués. L'évaluateur et son mandant peuvent donc conclure une entente de nature publique ou encore une autre à caractère privé. II arrive aussi que cette entente soit un mélange des deux, c'est-à-dire qu'elle comporte des aspects publics et d'autres privés. La portée de l'évaluation et de ses résultats étant directement conséquente de l'une ou l'autre de ces situations, l'évaluateur et le mandant ont donc avantage à préciser contractuellement ce qui est public dans le mandat et ce qui revêt un caractère strictement confidentiel et privé.

De plus, par le biais de ses services et de son expertise, l'évaluateur fournit aux décideurs des informations permettant de prendre des décisions. Ces informations peuvent elles aussi posséder un caractère public ou privé qui sera déterminé en grande partie par les particularités du milieu et la nature du mandat. Certaines de ces informations peuvent se révéler a priori confidentielles tandis que d'autres relèvent essentiellement de l'intérêt public. II apparaît donc primordial que l'évaluateur fasse, avant d'entreprendre l'évaluation, une "épuration » des différentes informations à recueillir afin d'offrir aux participants les garanties nécessaires à la protection de leur intégrité.

Tout cela constitue pour l'évaluateur d'éventuelles sources de problèmes s'il ne clarifie pas ces aspects avant d'entreprendre l'évaluation. Il doit donc prendre le temps d'analyser tout le contexte privé et public du mandat et des informations à recueillir. Par ses fonctions et son rôle, l'évaluateur est d'une part redevable à son 
mandant (contractuellement et moralement) mais il l'est aussi (moralement) face aux participants et à l'évaluation. Cette analyse lui permettra par la suite de clarifier la situation auprès des parties concernées et ainsi d'apporter à tous des garanties basées sur des considérations éthiques et déontologiques.

\section{Précisions sur le mandat}

Dans l'ensemble, le mandant est le maître d'œuvre de l'évaluation. II revient cependant à l'évaluateur la responsabilité de définir et de structurer tout le «design " philosophique et opérationnel de l'activité. D'après Adelman (1980), cela va même jusqu'à déterminer les conditions et le cadre de l'entente conformément aux besoins et aux attentes des gens du milieu. L'expérience aidant, l'évaluateur verra à faire consigner au contrat le maximum de clauses afin de réduire au minimum les sources de problèmes. Dans un tel document, nous devrions retrouver des aspects aussi importants que :

- la nature précise du mandat incluant tout ce que l'on attend de l'évaluateur;

- l'identification du mandant à qui l'évaluateur doit rendre compte du travail accompli;

- qui sont les décideurs et quels mécanismes seront utilisés pour résoudre les problèmes;

- l'identification des auditoires cibles ainsi que les ententes pouvant être conclues entre les diverses parties;

- des précisions sur les délais à respecter et les coûts engagés ou prévus;

- les autorisations pertinentes quant à l'utilisation et au cheminement des informations ainsi qu'à la production de rapports d'évaluation;

- tout autre élément qui pourrait devenir un obstacle à la conduite de l'évaluation ou porter à confusion dans diverses situations.

En procédant de la sorte, l'évaluateur contribue à déterminer des conditions d'intervention essentielles au bon déroulement de l'évaluation auxquelles il pourra se référer à tout moment dans ses relations professionnelles et humaines avec les gens du milieu. De plus, pour que de telles conditions s'inscrivent dans un contexte d'intervention pouvant être qualifié d'éthique, elles doivent s'inspirer de principes et de standards professionnels de haut niveau, eux-mêmes issus d'un modèle de conduite basé sur une morale reconnue et acceptée par les divers intervenants en cause. 


\section{Nécessité de disposer d'un référentiel de conduite professionnelle}

L'identification de principes et de standards professionnels a comme but ultime de guider les praticiens d'une discipline, ici l'évaluateur, dans les jugements et dans les actes qu'ils font. Ils servent aussi de points de référence à tous les intervenants de l'évaluation afin d'apprécier la conduite du spécialiste. II apparaît donc important et essentiel d'identifier des principes et des standards qui regroupent des conduites et des comportements types reconnus comme acceptables, moraux et susceptibles de répondre aux exigences de l'évaluation et des divers intervenants.

Une fois regroupés et consignés dans un guide de conduite appelé code de déontologie, ces conduites et ces comportements types imposent souvent au spécialiste la double condition d'être à la fois tributaire et gardien de la morale à appliquer dans l'exercice de sa profession. En maintes occasions, cette dualité peut s'avérer problématique pour le professionnel et ainsi provoquer des situations qui se transforment rapidement en véritables cas de conscience pour l'évaluateur. Comme Cook le décrit au chapitre 7 du volume de Selltiz (1977), les demandes d'informations de tierces personnes - le plus souvent sous des aspects intimes de la vie privée des gens - auprès des spécialistes sont fréquentes. De ce fait, elles placent souvent ceux-ci dans des situations conflictuelles. Alors, comment réagir dans de telles situations ? L'évaluateur serait-il tenté d'utiliser des moyens indirects et cachés afin d'obtenir des informations qu'il ne pourrait avoir autrement ? Les buts avoués de l'évaluation sont-ils réellement ceux poursuivis ? Les données recueillies révèlent-elles des informations qui peuvent être divulguées à d'autres personnes que celles directement concernées ? Voilà seulement quelques questions problématiques qui mettent en cause toute l'approche éthique de l'évaluateur et qui sont fondamentales tant pour la réputation de ce spécialiste que pour le déroulement de l'évaluation. L'identification de principes et de standards devient, dans ce contexte, un moyen efficace pour trouver des solutions préventives visant à protéger la crédibilité professionnelle de l'évaluateur et celle de l'évaluation.

\section{Les sources de problèmes éthiques}

Comme le mentionne Smith (1985) : " Evaluators, having been trained primarily as researchers, have little formal preparation for or experience in dealing with problems of competing values or in deciding which actions are moral and which not (...). But how do moral problems arise in evaluation practice, and what is the nature of these 
problems ? » (p. 5). Pour répondre aux deux volets de cette question, l'auteur dégage six causes (qu'il qualifie de traits distinctifs des problèmes) qui se retrouvent individuellement ou simultanément dans les problèmes moraux rencontrés par les évaluateurs :

1. pour un seul problème, la complexité et la variété des interactions entre les intervenants créent de multiples conditions à respecter afin d'identifier et d'adopter des comportements convenables ou moraux. Donc, chaque problème possède une gamme de considérations conflictuelles;

2. les problèmes moraux peuvent être soulevés par des préoccupations personnelles ou professionnelles;

3. la confrontation des valeurs personnelles, collectives et professionnelles est fréquemment une source de problèmes entre les parties;

4. les questions morales mises en cause peuvent se rapporter à l'évaluation elle-même [méthodologie, procédures, techniques, etc.] aux motifs et aux buts de l'évaluation;

5. la compréhension des problèmes moraux requiert une vaste perspective d'analyse laspects : historique, philosophique, psychologique, organisationnel, etc.]. Limiter cette perspective nuit à l'identification des causes problème(s) et entretient les mésententes;

6. la sensibilisation aux solutions envisagées [issues morales] n'est pas suffisante pour régler un problème. II faut aussi offrir des garanties acceptables pour tous.

L'auteur précise que cette liste de causes (traits distinctifs) ne constitue qu'un point de départ pour comprendre les problèmes moraux et ainsi permettre à l'évaluateur de s'orienter vers la résolution de ces problèmes. À cette fin, il recommande de procéder à l'analyse a) de la nature, de la fréquence et de la sévérité d'un problème et b) des valeurs personnelles, collectives et professionnelles mises en cause, pour ensuite identifier des solutions techniques et non techniques permettant de dénouer la situation conflictuelle.

\section{L'analyse et la résolution de problèmes éthiques}

Pour Brown et Newman (1985), l'analyse et la résolution de problèmes éthiques passent obligatoirement par la définition et l'utilisation de principes et de standards de haut niveau. Idéalement, ces derniers devraient être consignés dans un guide auquel le spécialiste peut se référer à tout moment et trouver réponse à ses interrogations. Cependant, il n'en est pas ainsi en évaluation : l'évaluateur doit se définir ou emprunter à d'autres domaines des principes et des lignes 
de conduite. C'est pourquoi les auteurs suggèrent une démarche basée sur l'utilisation de trois questions permettant d'analyser et de résoudre les problèmes moraux :

- Quels sont les principes et les standards qui servent à guider les pratiques utilisées par l'évaluateur dans ce contexte précis ?

- Les problèmes rencontrés mettent-ils en cause l'application de ces principes et de ces standards ?

- Si oui, quels changements doit-on apporter pour que la relation entre la situation et l'utilisation de ces principes et de ces standards soit compatible?

Ces trois questions permettent à l'évaluateur a) d'identifier des lignes de conduite pertinentes à une situation, $b$ ) de vérifier, si le problème en est un d'ordre moral, l'adéquation des principes et des standards retenus, et c) d'apporter des correctifs pour que la conduite de l'évaluation se fasse dans les conditions les plus morales ou éthiques possibles.

Dans un ordre d'idées similaire, les auteurs Paradise et Siegelwaks cités par Kottler (1982) proposent un modèle plus formel pour résoudre les problèmes moraux comme les confrontations ou les mésententes qui surviennent dans un groupe de travail. Six étapes sont requises :

1. Cerner de façon précise toutes les causes du problème ou du dilemme.

2. Identifier une ligne de conduite éthique (principes et standards) pour aider à dénouer la situation.

3. Générer des actions possibles et probables.

4. Considérer les conséquences potentielles de chaque action.

5. Choisir la meilleure action conformément à la ligne de conduite retenue.

6. Vérifier l'obtention des résultats attendus (et reprendre le processus si nécessaire).

Procéder de cette façon, selon Tennyson et Storm (1986), c'est faire preuve de responsabilité. Cela permet d'assainir les relations interpersonnelles et de créer un climat de confiance essentiel à la conduite de l'évaluation. Ils proposent aussi comme solution aux problèmes éthiques a) d'étudier les concepts/valeurs des autres et d'en " tester " la validité, $b$ ) d'exposer nos propres concepts/valeurs et de vérifier tant les concordances que les différences, c) d'établir des modèles fonctionnels pour résoudre les problèmes pratiques. L'adoption de telles démarches dénote, chez un spécialiste, un sens empathique indiscutable et cela contribue aussi à lui donner des outils indispensables pour prendre des décisions éclairées et éthiques. 


\section{Les principes et les valeurs à considérer}

À ce jour, peu de travaux ont été menés pour établir des principes en évaluation. Les efforts de plusieurs auteurs et organismes ont surtout porté sur la définition de standards servant à guider la pratique de l'évaluation. Comme le soulignent Brown et Newman (1985) :

Undoubtedly, ethical considerations guided the evolvement of these standards, but these considerations were not made explicit. [...] Standards usually provide guidelines for minimal practices, whereas adherence to ethical principles provides a model for ideal practices - at least the potential for consideration of the ideal seems more likely to follow from ethical principles than from standards for practice. The two, standards and ethical principles, are unlikely to be mutually exclusive, but ethical principles are likely to be broader in scope. (p. 2)

Ils ajoutent que, la résolution de problèmes éthiques ne pouvant s'effectuer qu'en fonction d'une échelle de valeurs, le spécialiste n'a d'autre choix que de s'appuyer sur des principes moraux. Dans cette ligne de pensée, les standards servent à réduire au minimum les sources de problèmes, tandis que les principes éthiques vont au-delà de la prévention : ils servent à résoudre les problèmes. Même si la définition de standards représente une étape importante dans le processus de professionnalisation de l'évaluation, il leur apparaît plus approprié de développer un modèle de conduite basé sur des principes éthiques plutôt que sur des standards.

Comme nous l'avons vu précédemment, l'éthique est la science qui s'intéresse à la morale et cette dernière constitue l'ensemble des valeurs et des règles de conduite reconnues comme normes. Pour développer un modèle de conduite considéré comme éthique, il faut donc faire l'identification de normes (appelées principes) qui considèrent l'ensemble des valeurs et des règles de conduite des intervenants engagés dans l'évaluation. Pour cette étape du développement d'un modèle, précisons qu'ii ne s'agit pas de dresser une liste exhaustive de toutes les valeurs en cause. Bien au contraire, la démarche consiste à trouver des normes acceptables pour tous ou, si l'on préfère, des principes communs à tous les intervenants.

Zusman et Bissonnette (1973) ont relevé cinq catégories de valeurs affectant la conduite d'une évaluation. À celles-ci nous jugeons à propos d'ajouter deux autres catégories, soit les sixième et septième de la liste ci-après :

1. les valeurs personnelles de l'évaluateur;

2. les valeurs du mandant;

3. les valeurs des personnes qui participent à l'évaluation;

4. les valeurs de l'institution (ou du programme); 
5. les valeurs de l'agence qui produit les services professionnels;

6. les valeurs des auditoires cibles;

7. les valeurs professionnelles (évaluateur et autres spécialistes).

\section{Les principes éthiques fondamentaux}

Dans leur analyse de la conduite de l'évaluateur et des standards à respecter, Brown et Newman (1985) se reportent aux cinq principes éthiques de base énoncés par Kitchener (1984) :

\section{L'autonomie}

Ce principe comporte deux aspects. Le premier concerne le droit à la liberté de choix et d'action pour tous (y compris l'évaluateur). Le suivant porte sur la reconnaissance du droit des autres à l'autonomie, c'est-à-dire la non-interférence sur ce droit. De plus, les auteurs incluent dans ce principe tout ce qui concerne la prise de décision, la collaboration et la présentation de rapports pertinents et compréhensibles.

\section{La prévoyance}

Ce principe s'oriente principalement sur les torts qui peuvent être causés aux autres par des intentions de toutes sortes et sur les risques que ces personnes peuvent courir. Faire preuve de prévoyance, c'est aussi ne pas recueillir des données inutiles et respecter les budgets. Bref, le spécialiste qui respecte ce principe s'intéresse aux conséquences potentielles de l'évaluation sur les gens du milieu et donne des avis relevant de sa compétence ou prévoit la collaboration d'autres professionnels.

\section{La bienfaisance}

Ce troisième principe porte sur l'apport d'aspects positifs pour le bien-être des personnes. Les auteurs parlent d'aide au client (mandant) pour déterminer et comprendre où sont les sources $d^{\prime}$ information, comment créer un climat de confiance entre les intervenants pour la collecte des informations, fournir et traiter le maximum de données en fonction des besoins identifiés, proposer des solutions pour résoudre les problèmes, etc. Bref, indiquer aux diverses personnes concernées par l'évaluation des voies pour assurer le bien-être de tous. 


\section{La justice}

Faire preuve de justice dans une évaluation consiste essentiellement à traiter toutes les personnes de la même façon sans égard à certaines particularités comme la race, le sexe, le statut et autres. D'après les auteurs, ce principe est l'un des plus importants en évaluation, car il est intimement lié aux actes posés et à leurs conséquences. La justice permet notamment de garantir le respect des droits individuels ou collectifs, de prévoir que les auditoires seront informés et consultés, et elle favorise l'établissement d'un climat de confiance.

\section{La fidélitélloyauté}

Ce dernier principe porte sur un aspect fondamental et indispensable en éthique : I'engagement moral de l'évaluateur. Être fidèle, pour ce dernier, veut simplement dire être loyal envers ses engagements et respecter ses promesses. Pour éviter toute ambiguïté sur ce principe, l'évaluateur doit de toute évidence définir le cadre de l'intervention avant d'entreprendre l'évaluation. Une fois ce cadre défini, les engagements tant moraux que contractuels de ce spécialiste lui serviront de balises pour fournir un rendement maximal et pour respecter les attentes du mandant aussi bien que celles des autres intervenants.

Bien que ces principes soient suffisamment développés pour être considérés comme des principes de base, les auteurs font remarquer qu'ils laissent malgré tout place à de l'interprétation dans certaines situations. De plus, ajoutons que cette typologie des principes fondamentaux ne permet pas de résoudre des problèmes précis en éthique. Tout au mieux, elle permet à l'évaluateur de s'orienter vers une des attitudes préventives des problèmes éthiques. Qui plus est, lorsqu'on situe ces principes de base par rapport a) aux rôles de l'évaluateur, du mandant et des autres intervenants, $b$ ) aux besoins et aux attentes de toutes les parties, et $c$ ) aux techniques et à la procédure utilisée, les interactions qui se créent entre 1) les principes euxmêmes, 2) ces nouvelles variables, et 3) les principes et les variables prennent des proportions exponentielles et deviennent un nouveau problème à résoudre. Pour éviter une telle situation, il nous apparaît pertinent de développer une approche où chacun de ces principes fondamentaux est appuyé par des principes de soutien.

\section{Les principes de soutien}

Comme les écrits sur le sujet décrivent abondamment les principes à respecter en éthique, nous nous contenterons ici de citer les 
principes (au nombre de quatorze) qui semblent faire l'objet d'une préoccupation constante chez une multitude d'auteurs. II n'y a aucun ordre de priorité ou d'importance dans cette énumération de principes, car ils servent à soutenir les principes fondamentaux. De ce fait, vouloir établir une liste détaillée de ceux-ci et en faire une hiérarchisation semble aberrant et inutile, car chaque situation problématique demande en soi qu'un principe de base soit appuyé par un ou plusieurs principes de soutien.

Voici donc cette liste non exhaustive de principes de soutien :

- Légalité dans I'action;

- honnêteté;

- indépendance et autodétermination;

- respect sous toutes ses formes;
- disponibilité;

- confidentialité et secret professionnel;

— dignité;

— diligence;

- discrétion;
- bienséance;

- impartialité;

- désintéressement;

- intégrité;

- responsabilité.

Plusieurs autres principes pourraient se greffer à cette liste, de même qu'un principe de base pourrait devenir un principe de soutien pour un autre principe fondamental. Cela ne tend qu'à montrer la complexité des échelles de valeurs et de l'agencement qu'on peut en faire pour définir un modèle de conduite. Pour éviter de s'embourber dans des exemples aussi complexes que fastidieux, reportons-nous au schéma qui suit pour visualiser une façon de définir un modèle de conduite à partir des principes fondamentaux en éthique. Au départ, il existe une situation à laquelle l'évaluateur doit faire face. En utilisant un des cinq principes de base, ce dernier décortique la situation selon trois perspectives : les aspects techniques de la situation, les aspects philosophiques et les considérations psychologiques de cette situation. Pour chacune de ces perspectives, il dégage des principes de soutien qui permettent, une fois regroupés, de définir une conduite éthique. Si tel n'était pas le cas, il faudrait alors retourner à l'identification d'un autre principe de base et reprendre le processus jusqu'à ce qu'un modèle de conduite éthique soit déterminé pour cette situation.

Cette approche offre, selon nous, plusieurs avantages pour définir une conduite professionnelle éthique. En effet, aborder les principes éthiques dans une perspective d'ensemble revient à se précipiter dans une analyse inextricable des interactions de ces principes. Par contre, l'identification de principes de base comme ceux de Kitchener (1984) permet de situer la conduite de l'évaluateur dans un cadre général qualifié de moral. Toutefois, ceux-ci n'apparaissent pas suffisants pour définir une conduite éthique et l'ajout d'autres prin- 
cipes à cette typologie risque de rendre son utilisation trop ardue. II faut donc utiliser des principes de soutien. Par ailleurs, la diversité des situations rencontrées par les évaluateurs ne permet pas de circonscrire une série de principes de soutien et de se limiter à l'utilisation de ces derniers. Chaque évaluation apporte des situations particulières qui demandent l'utilisation de principes tout aussi particuliers. L'évaluateur doit donc utiliser une approche lui permettant de considérer les principes des divers milieux où il évolue tout comme ceux des intervenants engagés dans l'évaluation. L'utilisation de principes de soutien répond à cette exigence de la pratique de cette profession.

De plus, si l'on se reporte aux résistances rencontrées en évaluation et à la nature des divers problèmes, il apparaît que chaque situation peut être abordée selon les trois perspectives précitées. L'identification de principes de soutien pour chacune d'elles, associée à un processus d'analyse et de résolution de problèmes comme celui de Kottler précédemment cité, permet à l'évaluateur de résoudre les divers problèmes d'une situation qui, autrement, pourraient lui paraître difficiles à surmonter. Pour toutes ces raisons, il faut offrir à l'évaluateur une approche souple de définition de conduites et de comportements éthiques comme celle proposée au schéma de la page suivante. II ne reste plus alors à l'évaluateur qu'à définir les standards qui se rattachent à ces principes de soutien.

\section{Les standards}

Le concept de standard s'associe à celui de déontologie dans le processus d'élaboration d'un modèle de conduite. Ce processus se définit comme étant une règle établie, reconnue et fixée à l'intérieur d'un modèle. Dans la foulée de Brown et Newman (1985), nous pouvons y ajouter la notion de caractère minimal que véhicule un standard et la notion de types de standards identifiables. Précédemment, nous avons signalé trois perspectives pour chaque situation : les aspects techniques, philosophiques et psychologiques. À chacune de ces perspectives correspondaient des principes de soutien. Un standard étant une règle minimale associée à un devoir (lui-même défini par un principe), nous obtenons alors par les principes de soutien des standards dits techniques, philosophiques et psychologiques. Cependant, les standards philosophiques et psychologiques peuvent être regroupés sous une même appellation, car ils servent à définir les rapports entre les individus. Cela donnera finalement deux types de standards : les techniques et les comportementaux. La précédente définition peut alors se reformuler ainsi : un standard est une règle minimale, technique ou comportementale, qui est reconnue et fixée à l'intérieur d'un modèle. 


\section{Schéma : Utilisation des principes pour définir un modèle de conduite}

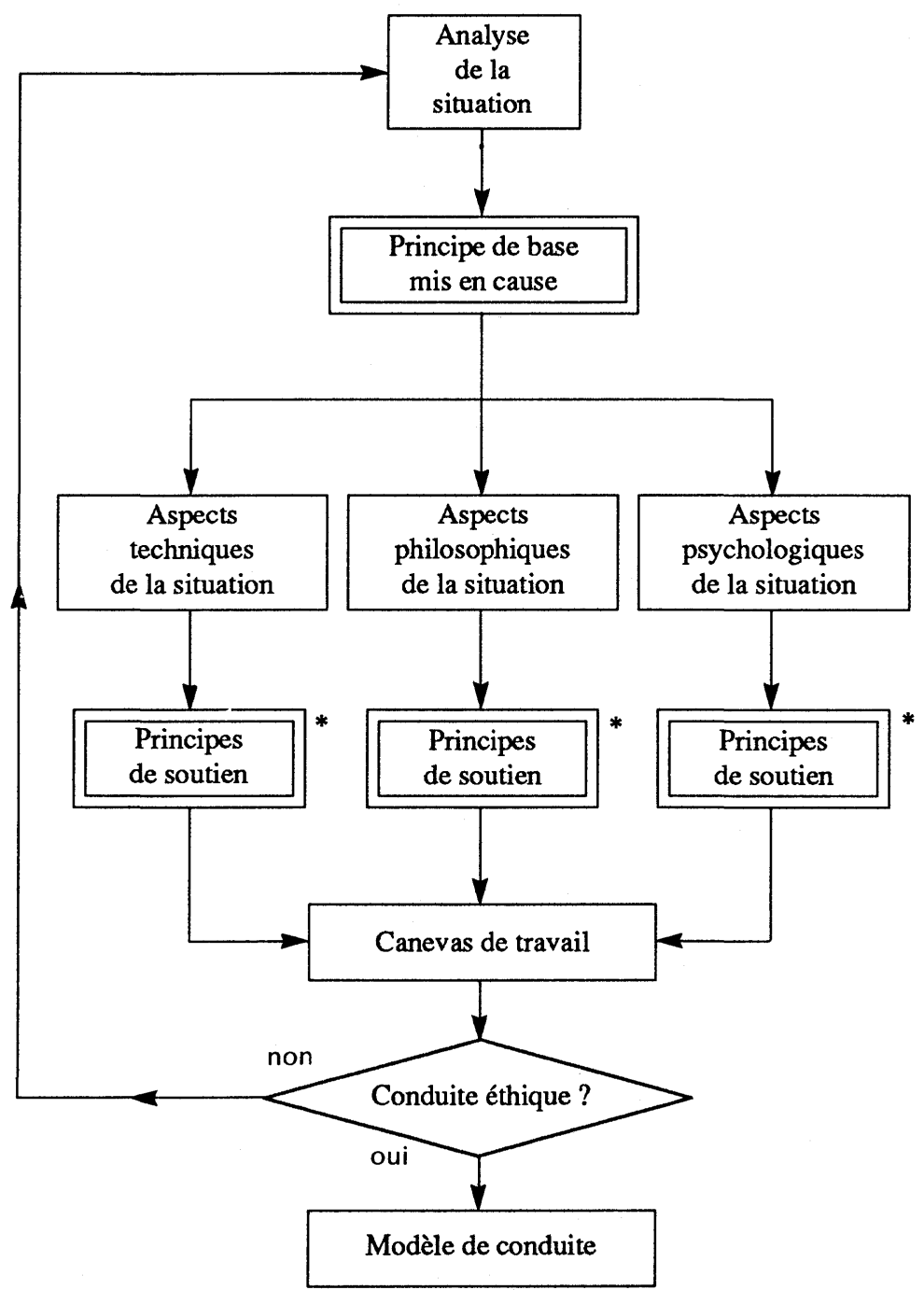

* Parallèlement, des standards peuvent être identifiés pour les principes de soutien. 


\section{Les études sur les standards}

Dans une collaboration à une publication de Rossi (1982), Stufflebeam fait la comparaison entre les standards du Joint Committee on Standard of Educational Evaluation (1981) et ceux de l'Evaluation Research Society (1982). Quoiqu'il note des différences entre les deux études, il souligne que les deux ensembles de standards sont en accord à bien des égards et que leur association serait profitable pour l'avancement de la profession d'évaluateur. D'autre part, Brown et Newman (1985) se sont aussi intéressés à ces deux classifications de standards, mais en les mettant cette fois en relation avec les principes de base de Kitchener (1984) énoncés précédemment. Bien qu'ils considèrent que ces deux études représentent un pas important pour la professionnalisation de l'évaluation, ils notent que certains principes fondamentaux ne sont pas couverts par les standards. Selon eux, il faudrait développer d'autres standards et porter une sérieuse attention aux principes de l'autonomie et de la fidélité/loyauté. Certes, soulignent-ils, certains de ces standards sous-tendent ces principes! Mais que se passera-t-il lorsqu'un évaluateur aura à résoudre un problème portant, par exemple, sur la justice et la fidélité ? Ou encore à l'occasion de tout autre conflit demandant l'intervention de plusieurs principes?

C'est en réponse à ces interrogations que nous avons adopté l'approche des principes fondamentaux et des principes de soutien illustrée au schéma déjà mentionné. Bien que des efforts louables et non négligeables aient été faits pour identifier des standards, des analyses comme celles de Brown et Newman (1985), Dockrell (1984), Raven (1984) et quelques autres montrent les faiblesses d'un modèle de conduite centré sur les standards. Sans vouloir déprécier le travail fait en ce sens, il semble que cette approche s'apparente plutôt à une démarche réactionnelle provoquée par un vide, par une absence de règles éthiques de pratique. Comme le souligne Nadeau (1988) en rapportant les propos de Cordray (1982), " Ils (les standards) représentent une série de prescriptions pour les évaluateurs ainsi qu'un excellent canevas de travail pour les responsables du développement de programme. [...] Les standards peuvent être utilisés pour revoir et corriger le rapport d'évaluation » (p. 162). La préoccupation majeure qu'illustrent ces propos apparaît être l'établissement d'un plan de travail, d'un modèle technique d'évaluation auquel on ajoute une série de prescriptions et de recommandations pour guider l'évaluateur d'un point de vue éthique. Certes, cela constitue une démarche intéressante pour cerner ce que doit être la conduite de ce spécialiste. Mais n'est-ce pas procéder à l'inverse que d'identifier des standards pour ensuite les faire correspondre à des principes ? Ne serait-il pas 
mieux de définir d'abord des principes éthiques pour ensuite leur associer des standards ? Cette seconde proposition permet d'éliminer plusieurs problèmes de l'approche basée sur les standards en favorisant avant tout le développement d'un cadre moral d'intervention auquel l'évaluateur identifie un modèle de conduite éthique et des standards à respecter (Dockrell, 1984; Raven, 1984; Brown et Newman, 1985). La préoccupation au centre de cette approche n'est plus le modèle d'exécution ou de réalisation de l'évaluation que l'on veut rendre éthique par des standards, mais plutôt l'établissement de balises morales servant à définir une conduite professionnelle pour tout ce qui a trait à l'évaluation. En conséquence, l'élaboration d'un modèle de conduite dit éthique s'effectue en considérant les attitudes et les comportements de l'évaluateur, de même qu'en s'intéressant à l'approche technique de l'évaluation. Voilà pourquoi nous croyons qu'il est préférable de développer d'abord des principes fondamentaux et de soutien pour ensuite identifier des standards techniques et des standards comportementaux qui découlent de ces principes, le tout devant évidemment être consigné dans un répertoire de règles de pratique habituellement appelé code de déontologie.

\section{Partie II Un code de déontologie pour l'évaluateur}

Étant donné la nature particulière des fonctions de l'évaluateur en éducation, santé et bien-être et la diversité des participants à une évaluation, les modèles consultés ne permettent pas de répondre entièrement aux préoccupations éthiques de cette profession. À cette fin, il nous faut bâtir un modèle qui tienne compte tant des principes fondamentaux et de soutien vus antérieurement que des standards identifiés précédemment. Pour cela, nous utiliserons la présentation et la formulation d'énoncés de principes que l'on retrouve dans certains codes de déontologie consultés à l'Office des professions et qui seraient applicables à la profession d'évaluateur. Outre ces énoncés de principes éthiques, nous retrouverons dans notre modèle les principes fondamentaux ainsi que les standards identifiés précédemment.

Ce modèle de code déontologique qui se veut applicable au secteur de l'éducation, santé et bien-être se divise en trois sections tout aussi distinctes que complémentaires. La première section regroupe, dans une perspective générale, les principes fondamentaux et les obligations qui doivent guider l'évaluateur dans l'exercice de ses fonctions. Elle sert donc à déterminer les règles d'ensemble à respecter pour définir un cadre éthique global. La seconde section porte 
plus spécifiquement sur les règles de conduite à privilégier par l'évaluateur et les divers intervenants. Ainsi, nous établirons des règles communes à l'ensemble des intervenants, de même que d'autres plus particulières à une catégorie de personnes. Finalement, la dernière section intitulée "Conduite technique de l'évaluation " situe les standards des études citées précédemment selon des thèmes ou des paramètres d'ensemble. Cette classification des standards dans le code résulte de la comparaison des standards faite par Stufflebeam (1982). En résumé, la présentation de ce code est progressive en ce sens que : 1) elle situe globalement le cadre éthique de la conduite de l'évaluateur, 2) elle précise le type de relations à privilégier entre les individus, et 3) elle situe la conduite de ce professionnel dans une perspective technique d'une évaluation.

\section{CODE DE DÉONTOLOGIE POUR L'ÉVALUATEUR}

\section{SECTION I : PRINCIPES ET OBLIGATIONS}

\subsection{Principes fondamentaux}

\subsubsection{Autonomie}

L'évaluateur doit reconnaître en tout temps le droit à la liberté de choix et d'action pour tous les intervenants appelés à participer à une activité d'évaluation.

\subsubsection{Prévoyance}

Dans l'exercice de sa profession, l'évaluateur doit tenir compte de l'ensemble des conséquences prévisibles pouvant affecter, directement ou indirectement, l'intégrité des personnes et la qualité de l'évaluation.

\subsubsection{Bienfaisance}

L'évaluateur doit appuyer toute mesure susceptible de sauvegarder ou d'améliorer la qualité de vie des personnes et du milieu d'intervention.

\subsubsection{Justice}

L'évaluateur doit traiter toutes les personnes de la même façon sans égard à la race, au sexe, au statut personnel et professionnel ou selon toute autre forme de discrimination.

\subsubsection{Fidélité / loyauté}

L'évaluateur doit respecter ses engagements et s'acquitter de ses promesses envers toute personne ou tout groupe de personnes. 


\subsection{Obligations générales}

\subsubsection{Bienséance}

L'évaluateur doit maintenir de hauts standards de conduite personnelle et professionnelle.

a) La conduite de l'évaluateur, quel que soit le milieu dans lequel il exerce sa profession, doit correspondre à des critères reconnus de bonne conduite.

b) L'évaluateur doit chercher à établir de saines relations entre les divers intervenants et favoriser un climat de coopération.

\subsubsection{Intégrité et crédibilité}

L'évaluateur doit agir selon de hauts standards d'intégrité, de crédibilité et d'impartialité.

a) L'évaluateur doit s'acquitter de ses devoirs professionnels avec intégrité et ne doit pas abuser de la confiance qu'on lui accorde.

b) La conduite de l'évaluateur doit être empreinte d'objectivité, de franchise et de droiture.

c) L'évaluateur ne peut ignorer volontairement un acte ou une activité découlant d'une malhonnêteté, d'une fraude, d'une supercherie ou d'une mauvaise représentation.

d) L'évaluateur doit être alerte et résister aux influences, aux pressions pouvant affecter son impartialité et sa crédibilité dans l'exercice de ses fonctions.

e) L'évaluateur ne peut exploiter à des fins personnelles des informations obtenues dans l'exercice de sa profession.

\subsubsection{Compétence}

L'évaluateur doit demeurer compétent et performer dans ses fonctions professionnelles.

a) Avant d'accepter un mandat, l'évaluateur doit tenir compte des limites de ses aptitudes, de ses connaissances, de son expérience ainsi que des moyens dont il dispose. II ne doit pas, notamment, entreprendre un travail d'évaluation pour lequel il n'est pas suffisamment préparé sans obtenir l'assistance nécessaire.

b) L'évaluateur doit s'abstenir d'intervenir sur des sujets qui ne relèvent pas de la compétence généralement reconnue à la profession.

\subsubsection{Services offerts}

L'évaluateur doit s'abstenir d'exercer dans des situations ou des états susceptibles de compromettre la qualité de ses services.

a) Avant de fournir ses services professionnels, l'évaluateur doit conclure une entente quant à l'ampleur et aux modalités des services requis et quant aux conditions de leur rémunération.

b) En plus des avis et des conseils, l'évaluateur doit fournir les explications nécessaires à la compréhension et à l'appréciation de services requis selon ce qui ressort de l'ensemble des faits qui ont été portés à sa connaissance. 
c) L'évaluateur doit éviter toute fausse représentation quant à l'efficacité de ses propres services et de ceux d'autres professionnels qu'il recommande ou qui sont appelés à collaborer à l'évaluation.

d) Sauf pour des motifs valables, l'évaluateur doit appuyer toute mesure susceptible d'améliorer la qualité et la disponibilité des services professionnels.

e) L'évaluateur doit informer le plus tôt possible les personnes concernées de toute erreur préjudiciable et difficilement réparable qu'il a commise en lui rendant un service professionnel.

f) L'évaluateur ne peut, sauf pour un motif juste et raisonnable, cesser d'agir pour le compte d'un client. Constituent notamment des motifs justes et raisonnables :

1. la perte de confiance de l'employeur ou d'un autre intervenant;

2. le fait que l'évaluateur soit en conflit d'intérêts ou dans un contexte tel que son indépendance professionnelle pourrait être mise en doute;

3. I'incitation, de la part d'un tiers, à l'accomplissement d'actes illégaux, injustes ou frauduleux;

4. la constatation, par l'évaluateur, de son incapacité ou de sa difficulté d'assurer la qualité des services professionnels.

g) Avant de cesser d'exercer ses fonctions, l'évaluateur doit faire parvenir à son client un préavis de délaissement dans un délai raisonnable et s'assurer que cette cessation de service n'est préjudiciable d'aucune façon à aucun des participants.

\subsubsection{Secret professionnel}

L'évaluateur doit respecter le secret de tout renseignement de nature confidentielle obtenu dans l'exercice de sa profession.

a) Lorsqu'un évaluateur demande à une personne de lui révéler des renseignements de nature confidentielle ou lorsqu'il permet que de tels renseignements lui soient confiés, il doit s'assurer que la personne est pleinement au courant du but de l'entrevue et des utilisations diverses qui peuvent être faites de ces renseignements.

b) L'évaluateur ne peut être relevé du secret professionnel qu'avec l'autorisation de l'employeur ou lorsque la loi l'ordonne.

c) L'évaluateur ne doit pas révéler qu'une personne a fait appel à ses services à moins que la nature du cas ne l'exige ou qu'il n'ait reçu l'autorisation (écrite) de la personne.

d) L'évaluateur doit éviter les conversations indiscrètes au sujet d'une personne et des services qui lui sont rendus.

e) L'évaluateur ne doit pas faire usage de renseignements de nature confidentielle à des fins autres que celles pour lesquelles ils lui avaient été confiés et, notamment, en vue d'obtenir directement ou indirectement un avantage pour lui-même ou pour autrui.

f) L'évaluateur doit respecter le droit de toute personne de prendre connaissance des documents qui la concernent dans tout dossier constitué à son sujet et d'obtenir une copie de ces documents. 


\subsection{6 Érudition, recherche et avancement de la profession}

L'évaluateur doit, dans la mesure du possible, aider au développement de sa profession par l'échange de ses connaissances et de son expérience avec ses confrères et les étudiants, et par sa participation à des cours ou des stages de formation et de perfectionnement.

a) L'évaluateur engagé dans une étude ou une recherche doit respecter les conventions qui s'appliquent aux méthodes d'investigation.

b) L'évaluateur doit considérer les conséquences possibles de la recherche sur le bien-être des individus.

c) L'évaluateur doit s'assurer du consentement volontaire des participants et garantir le respect de la dignité de ceux-ci et de la confidentialité des informations.

d) L'évaluateur consulté par un confrère doit fournir à ce dernier son opinion et ses recommandations dans le plus bref délai possible tout en préservant son indépendance professionnelle, ses engagements envers un tiers et son intégrité.

\section{SECTION II : CONDUITE DE L'ÉVALUATEUR ENVERS LES INTERVENANTS}

\subsection{Devoirs et obligations envers tous les intervenants}

\subsubsection{Climat de confiance}

L'évaluateur doit chercher à établir un climat de confiance mutuelle entre lui-même et les divers intervenants, tout comme entre les intervenants eux-mêmes.

a) L'évaluateur doit subordonner son intérêt personnel à celui de tous les intervenants, individuellement ou collectivement.

b) L'évaluateur doit mener ses entrevues ou ses rencontres de manière à respecter l'échelle de valeurs et les convictions personnelles ou collectives des intervenants.

c) L'évaluateur doit reconnaître en tout temps le droit aux intervenants de consulter un confrère, un membre d'une autre profession ou une autre personne compétente.

d) L'évaluateur doit apporter un soin raisonnable aux biens, aux documents et aux informations confiés à sa garde et il ne peut prêter ou utiliser ceux-ci à des fins autres que celles pour lesquelles ils lui ont été confiés.

e) L'évaluateur doit s'abstenir d'exprimer une opinion ou de donner des informations, des conseils contradictoires ou incomplets à quiconque. À cette fin, il doit chercher à avoir une connaissance complète des faits avant de donner une opinion ou un conseil.

\subsubsection{Disponibilité et diligence}

L'évaluateur doit faire preuve, dans l'exercice de sa profession, $d^{\prime}$ une disponibilité et $d^{\prime}$ une diligence raisonnables. 
a) L'évaluateur doit, lorsque sa présence est requise, se présenter ou se faire représenter, après en avoir avisé l'employeur, au temps et au lieu fixés.

b) $\mathrm{Si}$, pour une raison qui ne dépend pas de sa volonté, l'évaluateur ne peut se présenter ou se faire représenter à un rendez-vous ou à une rencontre fixée, il doit en donner avis aux parties intéressées.

c) L'évaluateur doit informer toute personne en faisant la demande du délai prévu pour l'exécution du mandat ou du cheminement fait.

d) L'évaluateur doit faire preuve d'objectivité et de désintéressement lorsque des personnes, autres que celles autorisées au mandat, lui demandent des informations.

e) L'évaluateur doit favoriser les mesures d'éducation et d'information pour l'ensemble des intervenants dans le domaine où il exerce.

\subsubsection{Indépendance et désintéressement}

L'évaluateur doit sauvegarder en tout temps son indépendance professionnelle et éviter toute situation où il serait en conflit d'intérêts.

a) Sans restreindre la généralité qui précède, un évaluateur est en conflit d'intérêts :

1. lorsque les intérêts en présence sont tels qu'il peut être porté à préférer certains d'entre eux à ceux d'une ou de plusieurs catégories d'intervenants ou que son jugement ou sa loyauté envers ceux-ci peuvent en être défavorablement affectés;

2. Iorsqu'il trouve dans l'acte professionnel pour lequel il agit comme conseiller un avantage personnel, direct ou indirect, actuel ou éventuel.

b) Dès qu'il constate qu'il se trouve dans une situation de conflit d'intérêts, l'évaluateur doit en aviser les parties intéressées et leur demander l'autorisation de continuer son mandat.

c) L'évaluateur ne doit révéler d'aucune façon les conclusions $d^{\prime}$ 'une évaluation à moins d'autorisation contraire.

\subsection{Devoirs et obligations envers l'employeur (mandant)}

\subsubsection{Responsabilités et engagements}

Nonobstant les devoirs et obligations envers les autres intervenants, l'évaluateur doit :

a) dès que possible, informer l'employeur de l'ampleur et des modalités du mandat que ce dernier lui a confié et il doit obtenir son accord à ce sujet;

b) adhérer aux politiques et aux procédures de l'employeur en pleine connaissance de cause;

c) s'assurer de la faisabilité et de la viabilité de l'évaluation et en informer l'employeur dans le plus-bref délai;

d) rendre compte à l'employeur lorsque celui-ci le requiert. 


\subsubsection{Fixation et paiement des honoraires}

L'évaluateur doit demander et accepter des honoraires justes et raisonnables.

a) Les honoraires sont réputés justes et raisonnables s'ils sont justifiés par les circonstances et proportionnés aux services rendus. L'évaluateur doit notamment tenir compte des facteurs suivants pour la fixation de ses honoraires :

1. le temps consacré à l'exécution des services professionnels;

2. la difficulté et l'importance des services;

3. la prestation de services inhabituels ou exigeant une compétence ou une célérité exceptionnelles.

b) L'évaluateur doit fournir à l'employeur toutes les explications nécessaires à la compréhension de son relevé d'honoraires et des modalités de paiement.

c) L'évaluateur doit prévenir l'employeur du coût approximatif de ses services et des débours nécessaires à l'exécution de son mandat.

d) L'évaluateur doit s'abstenir d'exiger d'avance le paiement de ses honoraires professionnels. II peut cependant exiger une avance pour couvrir le paiement des débours nécessaires à l'exécution de son mandat.

e) Pour un service donné, l'évaluateur ne doit accepter d'honoraires que d'une seule source, à moins d'entente explicite au contraire entre toutes les parties intéressées.

f) L'évaluateur ne peut percevoir des intérêts sur les comptes en souffrance qu'après en avoir avisé le débiteur. Les intérêts ainsi exigés doivent être d'un taux raisonnable.

g) Avant de recourir à des procédures judiciaires, l'évaluateur doit épuiser les autres moyens dont il dispose pour obtenir le paiement de ses honoraires.

h) Lorsqu'un évaluateur confie à une autre personne la perception de ses honoraires, il doit s'assurer dans la mesure du possible que celle-ci procède avec tact et mesure.

\subsection{Devoirs et obligations envers le décideur}

\subsubsection{Responsabilités et engagements}

Nonobstant les devoirs et obligations envers les autres intervenants, l'évaluateur doit :

a) clairement identifier la personne ou le groupe de personnes qui est responsable des décisions et de l'évaluation, avant d'entreprendre ses activités professionnelles;

b) informer le décideur dès que possible de l'ampleur et des modalités du mandat;

c) tout mettre en œuvre afin de faciliter l'accès à l'information au décideur et ainsi lui permettre $d^{\prime}$ exercer ses fonctions de façon juste et éclairée; 
d) sur demande du décideur, fournir à ce dernier tous les renseignements sur le déroulement de l'évaluation ainsi que tout document d'information ou rapport d'évaluation même s'il est réputé incomplet ou préliminaire;

e) ignorer toute intervention d'un tiers qui pourrait influer sur l'exécution de ses devoirs professionnels envers le décideur et, si nécessaire, aviser celui-ci d'une telle intervention;

f) rendre compte au décideur lorsque celui-ci le requiert.

\subsection{Devoirs et obligations envers la profession et les autres profes- sionnels}

\subsubsection{Responsabilités et engagements}

Nonobstant les devoirs et obligations envers les autres intervenants, l'évaluateur doit :

a) agir dans le respect de l'indépendance professionnelle de ses confrères et collègues;

b) faire preuve de courioisie, d'honnêteté et de bonne foi envers tout collègue de travail;

c) éviter de surprendre la bonne foi d'un autre professionnel ou de se rendre coupable envers lui d'un abus de confiance ou de procédés déloyaux. II ne doit pas, notamment, s'attribuer le mérite d'un travail qui revient à un confrère;

d) éviter les conversations indiscrètes au sujet d'un client et des services qui lui sont rendus;

e) promouvoir les valeurs, la morale et les missions de la profession d'évaluateur auprès des autres professionnels;

f) contribuer au développement et à l'avancement de la profession d'évaluateur;

g) favoriser la coexistence et l'épanouissement des autres professions rencontrées au cours d'une évaluation;

h) respecter la dignité professsionnelle. Est dérogatoire à la dignité professionnelle le fait de :

1. inciter quelqu'un de façon pressante ou répétée à rècourir à ses services professionnels;

2. tenter d'obtenir d'une personne un mandat qui, à sa connaissance, a déjà été confié à un confrère;

3. pactiser de quelque manière que ce soit avec toute personne pour se procurer des clients ou des affaires;

4. retarder indûment et volontairement l'exécution d'un mandat.

\subsection{Devoirs et obligations envers le public}

\subsubsection{Responsabilités et engagements}

Nonobstant les devoirs et obligations envers les autres intervenants, l'évaluateur doit :

a) favoriser des mesures d'éducation et d'information auprès du public; 
b) promouvoir le bien-être et le droit à l'information du public;

c) promouvoir la qualité et la disponibilité des services;

d) favoriser le développement et l'implantation de services;

e) tenir compte de l'impact social d'une évaluation et de ses conséquences ou implications sociales.

\section{SECTION III : CONDUITE TECHNIQUE DE L'ÉVALUATION}

\subsection{Formulation et négociation de l'évaluation}

Sans préjudice aux devoirs et aux obligations de toute autre personne, l'évaluateur doit :

a) définir les buts et les objectifs poursuivis par l'évaluation;

b) préciser la nature et les caractéristiques de l'objet évalué;

c) définir le type et l'étendue des activités d'évaluation;

d) procéder à la définition des auditoires, de leurs besoins, de leurs attentes et de leurs droits;

e) si nécessaire, favoriser la ratification d'ententes formelles entre divers intervenants et veiller au respect de ces conventions entre les parties;

f) décrire le ou les buts poursuivis par la procédure d'évaluation;

g) s'assurer de l'application et de la pertinence des méthodes et des techniques d'évaluation;

h) le cas échéant, exposer de façon précise les situations/conditions pouvant affecter la conduite de l'évaluation et les conclusions du ou des rapports;

i) prévoir des modalités de diffusion des rapports;

j) faire une juste et prudente estimation des coûts engendrés et des solutions souhaitables;

k) justifier les coûts liés à la collecte de l'information;

l) déterminer le nombre d'experts requis pour réaliser l'évaluation;

m) s'assurer que le personnel travaillant aux activités d'évaluation possède les qualifications professionnelles requises.

\subsection{Planification et structure de l'évaluation}

Sans préjudice aux devoirs et aux obligations de toute autre personne, l'évaluateur doit :

a) planifier et respecter un calendrier raisonnable pour toutes les activités prévues;

b) préparer et respecter un plan d'évaluation;

c) obtenir le consentement des parties intéressées sur la procédure et les effets à risques de l'évaluation;

d) favoriser la mise sur pied de mécanismes de coopération et de résolution de problèmes entre les parties; 
e) spécifier et justifier l'approche d'évaluation;

f) expliquer succinctement la méthode et les techniques qu'il compte employer;

g) utiliser des procédures et des instruments appropriés à l'objet d'évaluation;

h) s'assurer de la fidélité et de la validité des instruments de mesure;

i) prévoir, estimer les effets de l'évaluation.

\subsection{Collecte, traitement et analyse des informations}

Sans préjudice aux devoirs et aux obligations de toute autre personne, l'évaluateur doit :

a) respecter le plan de collecte des données et éviter tout écart du plan original sans le consentement des parties concernées;

b) dans la mesure du possible, reconnaître les sources d'erreurs;

c) vérifier la fidélité et la validité des mesures;

d) obtenir et produire une documentation complète des informations;

e) utiliser des sources d'information défendables;

f) protéger les données contre la perte, le vol ou la destruction;

g) reconnaître et expliquer les propriétés de l'analyse des données;

h) énumérer les postulats d'analyse;

i) mettre en évidence la pertinence des éléments ou unités d'analyse;

j) justifier l'analyse et produire un document d'explication sur la procédure d'analyse.

\subsection{Utilisation et divulgation des résultats}

Sans préjudice aux devoirs et aux obligations de toute autre personne, l'évaluateur doit :

a) respecter les engagements pris envers tous les participants;

b) faire une description complète, honnête et exhaustive de l'origine des résultats;

c) faire preuve de clarté dans la divulgation des résultats;

d) respecter la procédure d'utilisation et de divulgation des données;

e) rendre disponibles les résultats en temps opportun;

f) prévenir contre toute interprétation abusive ou erronée;

g) être attentif aux effets potentiels non prévus;

h) obtenir une ou des autorisations officielles pour publier, en tout ou en partie, les résultats. 


\section{Rapports : contenu et diffusion}

Sans préjudice aux devoirs et aux obligations de toute autre personne, l'évaluateur doit :

a) utiliser un langage clair et compréhensible pour tous;

b) à moins d'entente préalable contraire, fournir une rétroaction appropriée des résultats ou une copie du rapport aux auditoires ou aux personnes concernées par l'évaluation qui en font la demande;

c) organiser le contenu du rapport de façon à décrire l'ensemble de la démarche et ainsi couvrir tous les éléments essentiels à la compréhension de l'évaluation;

d) faire preuve d'objectivité dans le ou les rapports;

e) apporter les distinctions nécessaires entre les résultats de I'analyse et les recommandations;

f) apporter des conclusions et des recommandations justifiées;

g) fournir des informations sur les coûts et l'efficacité des options ou des recommandations faites;

h) clairement définir les limites de l'étude ou celles des études ultérieures.

\section{Conclusion}

Au début de ce document, nous faisions référence à la synergie existant entre l'éthique et la déontologie, pour l'élaboration d'un modèle de conduite. En fonction de cette synergie, nous avons apporté quelques éclaircissements sur la philosophie à privilégier dans une démarche professionnelle et sur les préconditions à considérer pour réaliser une évaluation éthique. Par la suite, nous avons abordé les concepts de principes fondamentaux et de soutien en éthique ainsi que celui des standards à satisfaire en évaluation. Toute cette démarche avait pour but l'élaboration d'un modèle de conduite à consigner dans un code de déontologie. Nous avons pu constater tout au long de ce cheminement que l'identification de règles et de devoirs met en cause une multitude de considérations et de réflexions qui peuvent parfois paraître, à certains, plus embarrassantes et exigeantes qu'utiles. Nous n'endossons évidemment pas cette opinion et nous croyons qu'une telle démarche ne peut que servir, à plus ou moins brève échéance, les intérêts de l'évaluateur et de la profession.

L'évaluation étant encore une jeune discipline, si nous la comparons à d'autres, il n'est donc pas étonnant de constater qu'il reste encore du chemin à faire avant qu'elle obtienne une reconnaissance professionnelle comparable à celle des professions archétypiques. Cependant, nous croyons que le processus de définition d'un modèle 
de conduite professionnel mène indéniablement les évaluateurs vers l'obtention ou la reconnaissance d'un statut professionnel. Certes, d'autres études ou recherches sur la définition de modèle de conduite peuvent s'avérer nécessaires pour en arriver à cela. Certaines porteront sur l'identification et l'analyse de principes en éthique, quelques autres tenteront d'associer à ces derniers les standards qui $s^{\prime} y$ rattachent; certaines recherches viseront l'amélioration technique ou philosophique de la conduite de l'évaluation, tandis que d'autres études porteront sur l'amélioration des modèles de conduite déjà existants. Bref, plusieurs études restent encore à réaliser, dans un avenir que nous souhaitons rapproché, pour obtenir une véritable professionnalisation du métier, de la profession d'évaluateur.

Entre temps, nous invitons les évaluateurs soucieux de pratiquer leur profession dans un contexte éthique à s'inpirer de modèles déjà utilisés dans d'autres disciplines ou encore à utiliser le code de déontologie que nous venons d'élaborer à cette fin pour eux. Comme le souligne Roberts dans le livre de Kottler (1982), les problèmes éthiques peuvent souvent se résoudre : $a$ ) en connaissant divers codes de déontologie, $b$ ) en étant prévoyant envers des dilemmes types avant d'entreprendre l'animation d'un groupe ou d'une évaluation, $c$ ) en consultant un expert d'une discipline appropriée lorsque des décisions difficiles sont envisagées, $d$ ) en demandant périodiquement la supervision d'un expert lorsque la situation l'exige, et e) en structurant et en utilisant un modèle de conduite [un code de déontologie] propre à la profession que l'on exerce. Avoir recours à ces quelques conseils de Roberts constitue, selon nous, plus qu'une approche de base pour développer une conduite professionnelle. Cela correspond plutôt à l'adoption d'une philosophie qui permet à tout professionnel de développer une chose importante et essentielle dans sa conduite : une conscience professionnelle éthique.

\section{Références bibliographiques}

Adelman, Clem. (1980). "Some Dilemmas of Institutional Evaluation and Their Relationship to Preconditions and Procedures ", Studies in Educational Evaluation, vol. 6, $\mathrm{n}^{\circ} 2:$ 165-183.

Brown, Robert D. et Dianna L. NeWman (1985). "Ethical Principles and Evaluator Roles ", Paper read at the Evaluation Network Annual Meeting : 1-12.

CORDRAY, D.S. (1982). Assessment of the Utility of the ERS Standards. Dans P.H. Rossi. Evaluation Research Society. Standards for Evaluation Practice. San Francisco, CA : Jossey-Bass Inc.

DOCKRELL, W.B. (1984). "Applicability of Standards for Evaluation of Educational Programs, Projects and Materials. In An International Setting : Qualitative Research ", Evaluation and Program Planning, vol. 7, n 4 : 347-354.

HowE, Elisabeth (1980). "Public Professions and the Private Model of Professionalism ", Social Work, vol. 25, n 3 : 179-191. 
INGLe, Marcus D. et Rudi Klauss (1980). Competency-Based Program Evaluation : A Contingency Approach, Evaluation and Program Planning, vol. 3, $n^{\circ} 4: 277-287$.

KILBURG, Richard (1980). " Metaphysics and Professional Resistance to Program Evaluation », Evaluation and Program Planning, vol. 3, $\mathrm{n}^{\circ} 3$ : 185-190.

KITCHENER, K.S. (1984). " Intuition, Critical Thinking, and Ethical Principles : The Foundation of Ethical Decisions in Counseling Psychology », The Counseling Psychologist, vol. 12, $\mathrm{n}^{\circ} 3$ : 3-55.

KOTTLER, Jeffrey A. (1982). "Ethical Issues in Group Work ", Journal for Specialists in Group Work, vol. 7, $\mathrm{n}^{\circ} 3: 140-186$.

LINCOLN, Yvonna S. (1985). The ERS Standards for Program Evaluation : "Guidance for a Fledging Profession ", Evaluation and Program Planning, vol. 8, $\mathrm{n}^{\circ} 3: 251-253$.

Mervin, Jack C. et Paul H. Wiener (1985). "Evaluation : A Profession?, Educational Evaluation and Policy Analysis, vol. 7, $\mathrm{n}^{\circ} 3$ : 253-259.

NADEAU, Marc-André (1988). L'évaluation de programme : théorie et pratique. Québec : Les Presses de l'Université Laval, $2^{\mathrm{e}}$ édition.

OFFICE DES PROFESSIONS DU QUÉBEC. Éditeur officiel du Québec. Gouvernement du Québec. Codes de déontologie consultés : comptables agréés, infirmières et infirmiers, ingénieurs, administrateurs agréés, conseillers d'orientation, conseillers en relations industrielles, évaluateurs agréés, psychologues, travailleurs sociaux, architectes et agronomes.

Paradise, Louis V. et Harriet E. Kramer (1981). "Ethics in Counseling ", Counseling and Values, vol. 25, $\mathrm{n}^{\circ} 4: 219-268$.

RAVEN, John (1984). "Some Limitations of the Standards ", Evaluation and Program Planning, vol. 7, $\mathrm{n}^{\circ} 4: 363-370$.

SelLtiz, C., L.S. WRIGHTSMAN et S.W. CoOK (1977). Les méthodes de recherche en sciences sociales. Montréal : Éditions HRW : 197-246.

SHAPIRO, Jonathan Z. (1985). Where We Are and Where We Need to Go. Educational Evaluation and Policy Analysis, vol. 7, $\mathrm{n}^{\circ} 3$ : 245-248.

Sieber, Joan E. et Nancy SANDERS (1978). "Ethical Problems in Program Evaluation : Roles, Not Models ", Evaluation and Program Planning, vol. 2, $\mathrm{n}^{\circ} 2$ : 117-120.

SMITH, Nick L. (1985). "Some Characteristics of Moral Problems in Evaluation Practice ", Evaluation and Program Planning, vol. 7, n 1 : 5-11.

StANFIELD, Jonathan et Nick L. SMITH (1985). "Management Consulting and Evaluation ", Evaluation and Program Planning, vol. 7, $\mathrm{n}^{\circ} 1$ : 87-93.

StUfFleBeAM, Daniel L. et al. (1974). L'évaluation en éducation et la prise de décision. Traduction de Jules Dumas, Victoriaville : Éditions NHP, 1980. (Édition américaine : Phi Delta Kappa, 1974).

StufflebeAM, Daniel L. (1982). Joint Committee on Standards for Evaluation. Standards for Evaluation of Educational Programs, Projects and Materials. New York, NY : McGraw Hill.

Tennyson, W. Wesley et Sharon M. Storm (1986). "Developing Responsibleness", Journal of Counseling Development, vol. 64, $n^{\circ} 5: 298-302$.

Windle, Charles et William NeIGHeR (1978). "Ethical Problems In Program Evaluation : Advice for Trapped Evaluators ", Evaluation and Program Planning, vol. $1, \mathrm{n}^{\circ} 2: 97-108$.

Zusman J. et R. BissonNetTE (1973). "The Case Against Evaluation (with some suggestions for improvement) ", International Journal of Mental Health, vol. 2 : 11-125. 